\title{
PROGRAMA PARA EL FORTALECIMIENTO DE LA AUTOESTIMA EN JUGADORAS DE FÚTBOL ADOLESCENTES
}

\section{PROGRAM FOR SELFSTEEM POTENTIATION AMONG TEEN FEMALE FOOTBALL PLAYERS}

\author{
Elmi José Odor Hurtado. EAE business School. España.
}

Pilar Portugal de la Cuerda. EAE business School. España.

Paula Salado García. EAE business School. España.

Olga Sanz Gómez. EAE business School. España.

Agradecemos este Trabajo a todas las personas que nos han posibilitado su desarrollo. A María Elena del Valle por su disposición y enseñanzas. Al Club Deportivo Pozoblanco por su predisposición y muy especialmente al equipo de jugadoras cadetes $B$ femenino del club, que lo han hecho, simplemente, posible.

\section{RESUMEN}

En este trabajo fin de máster se diseña y desarrolla una propuesta de intervención a través de un programa específico llamado 4Adelante, constituido por diferentes talleres y actividades, cuyo objetivo es la mejora de los niveles de autoestima en niñas adolescentes, de 10 a 15 años, jugadoras de fútbol en medio rural (Pozoblanco, Córdoba). El trabajo de investigación se centra en la autoestima, la importancia de trabajar su potenciación y en el entorno de niñas jugadoras de fútbol, que son cuestionadas en ocasiones por la práctica de este deporte y esto hace que puedan poner en duda su continuidad en el mismo. El propósito de este proyecto es el diseño de un programa de talleres y actividades que les haga trabajar y fortalecer la autoestima en el colectivo mencionado, con el fin de equiparar sus oportunidades y que puedan sentirse empoderadas a la hora de tomar sus decisiones, tanto a nivel deportivo como a nivel personal.

PALABRAS CLAVE: autoestima, deporte, fútbol femenino, adolescente, talleres.

\section{ABSTRACT}

In this article, an intervention proposal is designed and developed through a specific program called 4Adelante, made up of different workshops and activities, which goal is to improve self-esteem levels in adolescent soccer playing girls, from 10 to 15 years old, in rural areas (Pozoblanco, Córdoba). The research work focuses on the girl's self-esteem, the importance of working on their empowerment and in their environment, as soccer playing girls are sometimes questioned for their sport practices, making them question their continuity in such activities. The purpose of this project is to design a program of workshops and activities aimed at making them work and strengthen their self-esteem in the aforementioned group, in order to equalize their opportunities and make them feel empowered when making their decisions, both at a sports and personal level.

KEY WORDS: self-esteem, sport, women's soccer, teenagers, workshops. 


\section{Cómo citar el artículo:}

Odor Hurtado, E. J., Portugal de la Cuerda, P., Salado García, P. y Sanz Gómez, O. (2020). Programa para el fortalecimiento de la autoestima en jugadoras de fútbol adolescentes. Revista de Ciencias de la Comunicación e Información, 25(2), 157-182.

doi: http://doi.org/10.35742/rcci.2020.25(2).157-182

\section{INTRODUCCIÓN}

La adolescencia temprana se considera especialmente relevante para el desarrollo de la autoestima en el individuo. El propósito de este estudio es correlacionar el incremento de la autoestima con la aplicación de un programa de talleres digitales, en niñas adolescentes, jugadoras de fútbol. La adolescencia es una etapa que conlleva la vivencia de situaciones nuevas, no siempre fáciles, que suponen un desafío para la visión que los adolescentes tienen de sí mismos (Steinberg y Morris, 2001; Twenge y Campbell, 2001). El estudio de la autoestima en la mujer es una de las grandes tendencias en investigación, como es creciente también en los últimos tiempos, la práctica de deporte femenino en la disciplina del fútbol.

Según la encuesta realizada por ONU Mujeres (2018) sobre la igualdad de género en el deporte, los estereotipos de género en el deporte difundidos por los medios de comunicación y espacios educativos, fundamentalmente, influyen en la práctica deportiva de niños y niñas. La encuesta determina que la principal razón por la que las niñas abandonan el deporte y piensan que no es un espacio para ellas, es la falta de redes de apoyo y de espacios habilitados para que puedan potenciar sus capacidades deportivas. Este abandono es sin duda limitante para ellas como lo es la creencia de que no sea un espacio femenino y apto para su desarrollo como deportistas.

Este trabajo se centra en la autoestima y su posible potenciación, tomando como base varios de los pilares referidos por Branden, en el colectivo de deportistas femeninas, que practican un deporte realizado mayoritariamente por varones y en un momento vital, para el desarrollo de su autoestima, como es la adolescencia.

\section{PLANTEAMIENTO DEL PROBLEMA}

La autoestima engloba una serie de percepciones propias desarrolladas a lo largo del tiempo por cada individuo y que se relaciona estrechamente con las expectativas que tiene sobre sí mismo. Es un constructo medible en conexión con la idea que cada uno tiene de su propia valía. Una alta autoestima, aventaja a quien la posee, ya que le otorga la opción de sentirse valioso y facilita su desempeño, según la valía que el individuo cree disponer. Esta alta valía es la base de las personas con alta autoestima y se vincula al bienestar mental en deportistas, por su relación, entre otros factores, con la resiliencia. 
El objetivo de un deportista es conseguir sus metas, lo que está altamente relacionado con la capacidad que tiene de reconocer sus habilidades, capacidades y limitaciones. Para conseguir sus objetivos, las niñas jugadoras de fútbol deben tener actitudes y capacidades, que les permitan superar retos con éxito. Además, desarrollar habilidades y tener una fuerte creencia en sí mismas para permanecer y destacar en un ámbito tradicionalmente masculino, donde el cuestionamiento por género está muy extendido.

Existen diferentes estudios acerca del prejuicio hacia la mujer deportista, entre ellos el de Abbassi (2016), en el que mantiene que existe mayor discriminación hacia e género femenino, especialmente, por parte de los hombres que no realizan deportes de contacto. Según la encuesta realizada por ONU (2018) la mayoría de los niños y niñas tienen autoestima elevada. Las principales diferencias se manifiestan por el nivel socioeconómico y cuando se considera la variable discriminación en el deporte. En el último caso, las niñas son las principales afectadas.

\section{METODOLOGÍA DE LA INVESTIGACIÓN}

\subsection{Objetivos}

El presente trabajo se enmarca en el ámbito exploratorio en el que se pretende investigar la posible relación entre los niveles de autoestima y una intervención grupal a través de talleres digitales.

Para desarrollar esta investigación nos planteamos las siguientes cuestiones:

- ¿Qué nivel de autoestima tienen las jugadoras del equipo de fútbol cadete de Pozoblanco FC?

- ¿Una intervención a través de talleres digitales puede fortalecer la autoestima del grupo?

- ¿Cuál sería el nivel de autoestima de las jugadoras del equipo de fútbol cadete de Pozoblanco FC al finalizar la intervención?

\subsection{Objetivo general}

Tras plantear las cuestiones anteriores y habiendo realizado un trabajo de investigación al respecto, se plantea el objetivo general de este estudio: diseñar un programa de talleres para el fortalecimiento de la autoestima, orientado a jugadoras de fútbol adolescentes, y determinar si este incrementa el nivel de autoestima de las mismas.

\subsection{Objetivos específicos}

1. Diagnosticar el nivel de autoestima del equipo antes de la implementación del programa.

2. Aplicación del programa online diseñado: 4Adelante

3. Determinar el nivel de autoestima del equipo después de la implementación del programa. 


\subsection{Hipótesis específicas}

Las hipótesis específicas que nos han servido de guía en el proceso de investigación y nos dan orientación de cómo resolverla son:

1. Es importante conocer el punto de partida del nivel de autoestima, antes de llevar a cabo el programa de fortalecimiento de la autoestima.

2. La autoestima de las jugadoras se fortalecerá gracias a la aplicación de un programa de talleres. ¿No es igual que la general?

3. El conocimiento del nivel de autoestima tras la implementación del programa nos permitirá conocer los resultados de la aplicación del mismo.

\section{ESTRATEGIA DE MEDIDA}

La escala escogida para la realización de la medida de la autoestima es la de Escala de Autoestima Rosenberg (RSES), una de las más utilizadas en la investigación, traducida a 28 idiomas y utilizada y validada en más de 50 países. Es el instrumento psicométrico más utilizado para medir la autoestima actualmente, debido a su aplicabilidad, rapidez y sencillez.

Por otro lado, hemos podido adecuar con facilidad y fiabilidad esta escala a una herramienta digital como Survey Monkey, que se adapta muy bien a la edad de las integrantes del equipo (permitiendo su realización a través del móvil) y a la realidad social COVID-19, que nos ha llevado a la digitalización de todos los talleres y herramientas.

ESCALA DE AUTOESTIMA DE ROSEMBERG (RSE)

(Rosenberg , 1965; Atienza, Balaguer, \& Moreno, 2000)

Por favor, lee las frases que figuran a continuación y señala el nivel de acuerdo o desacuerdo, marcando con un aspa la alternativa elegida

\begin{tabular}{|c|c|c|c|c|c|}
\hline & & $\begin{array}{l}\text { Muy de } \\
\text { acuerdo }\end{array}$ & De acuerdo & \begin{tabular}{|c|} 
En \\
desacuerdo \\
\end{tabular} & $\begin{array}{c}\text { Muy en } \\
\text { desacuerdo }\end{array}$ \\
\hline 1 & Me siento una persona tan valiosa como las otras & & & & \\
\hline 2 & Creo que tengo algunas cualidades buenas & & & & \\
\hline 3 & Generalmente me inclino a pensar que soy un fracaso & & & & \\
\hline 4 & Soy capaz de hacer las cosas tan bien como los demás & & & & \\
\hline 5 & Ceo que no tengo mucho de lo que estar orgullosa & & & & \\
\hline 6 & Tengo una actitud positiva hacia mi misma & & & & \\
\hline 7 & En general me siento satisfecha conmigo misma & & & & \\
\hline 8 & Me gustaría tener más respeto por mi misma & & & & \\
\hline 9 & Realmente me siento inutil en algunas ocasiones & & & & \\
\hline 10 & A veces pienso que no sirvo para nada & & & & \\
\hline
\end{tabular}

Figura 1. Escala de Autoestima de Rosenberg. Fuente: Elaboración propia.

\section{MARCO TEÓRICO}

\subsection{Bases teóricas de la autoestima}

Desde William James (1890) considerado pionero en el constructo de la autoestima para la psicología, y que se aproxima a una conceptualización de la autoestima más 
cercana a la de hoy, hablando de que "La estima que sentimos por nosotros depende enteramente de lo que pretendamos ser y hacer" como es citado en Monbourquette, J. (2002).

El estudio de la autoestima se retoma con fuerza a mediados del siglo XX, cuando se publica la definición que hizo Rosenberg (1965) y que es citada por MolinaGarcía; Castillo \& Pablos (2007): "la manifestación de la actitud global (favorable o no) que una persona posee, en relación a su importancia y valía".

Abraham Maslow (1979), a través de su teoría de la pirámide y la motivación, describe la autoestima como una necesidad absoluta y real, que se manifiesta "en el respeto que le merecemos a otros, más que el renombre, la celebridad y la adulación". Nathaniel Branden (1999) afirma que se trata de: "La experiencia de ser aptos para la vida y para las necesidades" y la define (1995) como: "la confianza en nuestra capacidad de pensar, en nuestra capacidad de enfrentarnos a los desafíos básicos de la vida, la confianza en nuestro derecho a triunfar y a ser felices y el sentimiento de ser respetables, de ser dignos, y de tener derecho a afirmar nuestras necesidades y carencias, a alcanzar nuestros principios morales y a gozar del fruto de nuestros esfuerzos".

Catherine Cardinal (2007), escribió: "la autoestima es el aprender a confiar en uno mismo, confiar en nuestro instinto, el desarrollar una conexión con nuestro cuerpo y escuchar los mensajes que nos envía". Es decir, si podemos escuchar a nuestro interior vamos a poder tomar decisiones saludables. La autora defiende que: "una autoestima saludable es prestar atención a cómo nos hacen sentir las demás personas y luego elegir con quien estar", mientras que "la baja autoestima resulta cuando nos criticamos a nosotros mismos".

El médico psiquiatra y profesor de psiquiatría en la Universidad de New York, Luis Rojas Marcos (2007), en su libro "La autoestima: nuestra fuerza secreta", analiza los factores que determinan la idea de nuestro "yo", como la infancia, los valores culturales, el autocontrol, el odio a uno mismo, los estados depresivos, así como el papel de la autoestima en las relaciones con los demás.

Dos frases célebres de Jack Canfield (2008) sobre la autoestima son: "La autoestima se compone principalmente de dos cosas: sentirse digno de ser amado y sentirse capaz" y "Mayor autoestima produce un mayor éxito y más éxito produce autoestima más alta, por lo que mantiene en espiral hacia arriba".

La periodista Tamara Sánchez (2018), en su artículo "Definición, significado y tipos de autoestima" cita: "Lo que los demás ven en nosotras o, más bien lo que nosotras pensamos que los demás ven, es crucial para determinar nuestro grado de autoestima".

Tras adentrarnos en el estudio de la autoestima y para abordar nuestro trabajo de investigación, contemplamos la autoestima a través de las definiciones revisadas, como una conjugación de creencias, emociones, percepciones, pensamientos, actitudes y comportamientos dirigidos hacia uno mismo. Branden, quien inspira finalmente nuestro programa, la determina como la disposición a considerarse 
competente, frente a los desafíos básicos de la vida y sentirse merecedor de la felicidad.

\subsection{Factores que influyen en el desarrollo de la autoestima}

La autoestima es un constructo complejo, abstracto y, en ocasiones, complicado de medir. La formación de nuestra autoestima depende de muchos aspectos como: la educación, las experiencias vividas, el nivel de perfeccionismo, etc.

Para Coopersmith (1967) existen cuatro criterios influyentes en la estructuración de la autoestima en un individuo.

1. La significación: el grado en que el sujeto se siente cómodo y aceptado por las personas que son importantes para él.

2. La competencia o grado que creemos poseer para desempeñar una tarea que consideramos valiosa e importante.

3. La virtud o valoración moral o ética que hacemos de nosotros mismos.

4. El poder o capacidad que la persona cree tener para controlar su vida e influir en la vida de los demás.

McKay y Fanning (1999), señalan que las bases de la autoestima se encuentran en la educación recibida durante la infancia. Existe actualmente suficiente evidencia acerca de la importancia de su desarrollo en el contexto escolar y de su impacto en el rendimiento. Una buena autoestima favorece que la persona se sienta capaz, sienta que cuenta con los recursos para lograr esas metas, considerando la importancia de su esfuerzo y trabajo. Los factores que proponen son varios:

- El entorno familiar

- La escuela y la sociedad.

- La infancia.

\subsection{Niveles de la autoestima}

Coopersmith (1976), afirma que la autoestima puede presentarse en tres niveles, que se diferencian entre sí según el comportamiento de los individuos: alta, media o baja. Y se evidencia porque las personas experimentan las mismas situaciones de forma notablemente diferente, dado que cuentan con distintas expectativas sobre el futuro, reacciones afectivas y auto concepto.

Autoestima alta es el nivel deseable para que una persona logre sentirse satisfecha en la vida, sea consciente de su valía y de sus capacidades y pueda enfrentarse a los inconvenientes de forma resolutiva.

Los individuos con un nivel de autoestima media afirman que son personas que se caracterizan por presentar similitud con las que presentan alta autoestima, pero la evidencian en menor magnitud y, en otros casos, muestran conductas inadecuadas que reflejan dificultades en el auto concepto.

Las personas con un nivel de autoestima baja son individuos que muestran desánimo, depresión, aislamiento, se sienten poco atractivos, así como incapaces de expresarse y defenderse, pues sienten temor de provocar el enfado de los demás. Las personas con este nivel se consideran débiles para vencer sus 
deficiencias, permanecen aislados ante un grupo social determinado, son sensibles a la crítica y se encuentran preocupadas por problemas internos.

Eisenberg y Patterson (1981) describen las conductas relacionadas con la autoestima alta y la autoestima baja. Destacan: expectativas relacionadas con el futuro, asertividad, maneras de afrontar el estrés, asunción de riesgos, temor al fracaso y aspiraciones vitales.

Según refiere Branden (1995) algunas personas tienen el impulso para lograr sus objetivos, a pesar de poseer una autoimagen baja: como puede ser el caso del adicto al trabajo, que será muy productivo, pero porque se siente impulsado a demostrar sus méritos. A pesar de ello, serán menos eficaces y felices por ello.

\subsection{Pilares de la autoestima}

Nathaniel Branden (1995), en su libro "Los seis pilares de la autoestima", refleja que la autoestima es la disposición a considerarse competente frente a los desafíos de la vida (eficacia personal) y sentirse merecedor de la felicidad (respeto a uno mismo). En este sentido, concluye que, en la actualidad, la felicidad personal y la supervivencia económica se basan, en gran medida, en la forma de comprender y fomentar la autoestima.

Branden propone que la autoestima puede trabajarse y depende fundamentalmente de uno mismo. Para su desarrollo define los 6 pilares fundamentales sobre los que trabajar:

1. Autoconocimiento.

2. Auto aceptación.

3. Autorresponsabilidad.

4. Autoafirmación.

5. Auto propósito.

6. Integridad personal.

Branden propone que hay que actuar de manera consciente, es decir, independientemente de las vivencias del individuo, de su educación y otros factores. Según el autor siempre se puede trabajar para mejorar la autoestima.

Este planteamiento sirvió de base para el desarrollo del programa de fortalecimiento de la autoestima en jugadoras de fútbol adolescentes.

Diseñamos los talleres aplicando cuatro de estos pilares, reformulando su nomenclatura:

1. Autoconocimiento

2. Auto aceptación

3. Autor respeto

4. Autoconfianza

Consideramos que son los de mayor aplicabilidad y mejor comprensión por parte de la muestra seleccionada. Descartamos vivir con propósito e integridad personal por 
la complejidad para el desarrollo a través de los talleres dada la edad de los componentes de la muestra.

Con los 4 pilares seleccionados además del trabajo individual que supone cada pilar, podíamos establecer con el equipo, una cierta conexión entre ellos y con un orden en el desarrollo de los mismos.

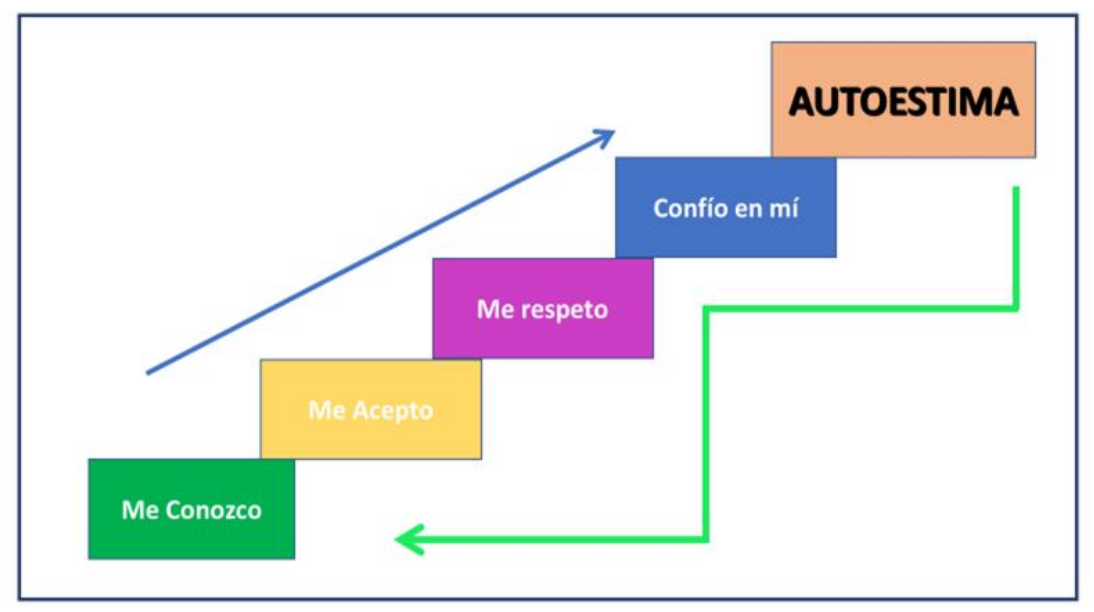

Figura. 2: Escalera de la autoestima.

Fuente: Elaboración propia.

\subsection{Importancia de la autoestima}

La autoestima consiste en tener una actitud positiva hacia uno mismo. Por lo tanto, la autoestima implica básicamente quererse y respetarse. Desarrollarla, trabajarla y mejorarla nos ayuda a sentirnos mejor, lo que influye en nuestro comportamiento.

Ana M. Bastida de Miguel (2010), expresa que: "Autoestima, es el concepto que tenemos de nuestra valía y se va formando en función de todos los pensamientos, sentimientos, sensaciones y experiencias que sobre nosotros mismos vamos incorporando a lo largo de nuestra vida. Todas las impresiones, evaluaciones y experiencias que vamos experimentando se van acumulando y van conformando un sentimiento positivo hacia nosotros mismos o, por el contrario, un incómodo sentimiento negativo por no ser lo que nos gustaría ser".

\subsubsection{La autoestima en el deporte}

La autoestima en el mundo del deporte tiene que ver con el grado en que el deportista consigue unos valores o estándares que considera «ideales». Cuanto mayor sea el gap entre lo que hace y consigue respecto a su ideal, más baja será la autoestima. $Y$ de manera inversa, cuanto más cerca esté de estos resultados o conductas ideales, más alta se situará su autoestima.

Castillo \& Pablos (2007), han concluido en sus investigaciones que el hecho de practicar deporte de forma regular afecta positivamente a los estados de ánimo y 
emociones que sentimos: alegría, miedo, patologías como la ansiedad y la depresión, además de mejorar la autoestima.

Se puede decir que una buena autoestima tiende a facilitar el rendimiento deportivo, (Molina; Chorot; Valiente \& Sandín, 2014). Por otra parte, el deporte influye directamente en el auto concepto físico que tiene cada persona de sí misma. De igual modo, se ha observado que la mayoría de las personas que tienen una autoestima elevada tienden a cuidar su organismo y su estado de salud (Espinoza; Rodríguez; Gálvez; Vargas \& Yáñez, 2011). Fernando Callejo Muñoz (2010), en su tesis concluyó: "Los estados de ánimo permanecen relativamente estables antes de cada uno de los diez partidos disputados en cada uno de los equipos valuados".

Con posterioridad, Hurtado Girón, Shaaron Getzabel (2014) en su tesis titulada "Nivel de autoestima y éxito deportivo en jóvenes que practican taekwondo" realizada en la Universidad Rafael Landivar, llegaron a la conclusión de que "los jóvenes que poseen alta autoestima manejan buenos niveles de adaptación, son optimistas, tenaces, confían en sí mismos y son persistentes en la búsqueda de las metas deportivas. Lo que explica que hay una relación de correspondencia entre autoestima y éxito en la práctica deportiva”.

Joan Pere Roig Barceló (2015), en el trabajo final titulado "El deporte y la autoestima como factores de socialización e inclusión social" realizado en la Universidad de las Islas Baleares, concluyen: "el deporte influye favorablemente en la reeducación y la rehabilitación social de los sujetos, siempre a favor de su inclusión social y del aumento o potenciación de su autoestima".

\subsubsection{La autoestima en adolescentes}

La autoestima se refiere a los sentimientos que tienen las personas respecto a sí mismas, siendo consistente transituacional y transtemporalmente (Brown, Dutton y Cook, 2001), si bien su estabilidad varía en función del ciclo vital (Trzesniewski, Donnellan y Robins, 2003). A menudo se confunde el término autoestima con una opinión agrandada sobre uno mismo, sin embargo, es necesario que los adolescentes entiendan que la autoestima juega un papel fundamental para el crecimiento como personas y estudiantes.

Más allá de apreciar lo positivo y aceptar lo negativo de uno mismo, hay algo que fundamenta este concepto y sin lo cual, la autoestima se desmoronaría. Se trata, como indica Bonet (1997), de reconocer un principio fundamental, recordado por todos los psicoterapeutas humanistas: "Todo ser humano, sin excepción (incluyendo yo mismo), por el mero hecho de serlo, es digno del respeto incondicional de los demás y de sí mismo; merece que se le estime y que se estime".

Para evaluar la autoestima en los adolescentes se desarrolló una herramienta: La Escala de Autoestima de Rosenberg en el 1965: ésta incluye diez ítems basados en sentimientos de respeto y aceptación de uno mismo/a. La mitad de estos ítems están enunciados de manera positiva y la otra mitad en manera negativa. Es un instrumento unidimensional que se contesta en una escala de 4 alternativas, que va desde "muy de acuerdo" a "muy en desacuerdo". 
La pirámide de Maslow, por ejemplo, es un elemento que nos indica la importancia de satisfacer todos los valores de necesidades para estar en equilibrio; en el 4 escalón encontramos el reconocimiento, que se divide en dos niveles: por un lado, auto-reconocimiento y, por otro, la necesidad de reconocimiento de las demás personas (respeto, éxito, confianza, etc.).

\section{Pirámide de Maslow}

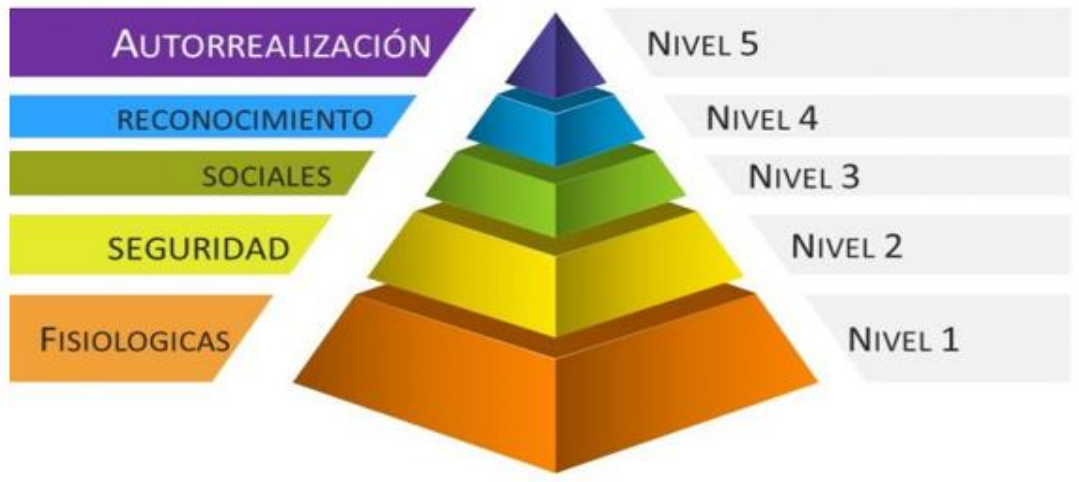

Figura. 3: Pirámide de Maslow.

Fuente: PiramidedeMaslow.net

Los adolescentes vienen de una etapa, la infancia, donde por lo general, el nivel de autoestima es muy alto y con los cambios que se producen en esta nueva etapa, tanto físicos como personales, ésta tiende a venirse abajo.

Branden (1995) expresa que este es un camino sinuoso y advierte que no es fácil llegar a la valoración propia a partir de una desvaloración que nos damos a nosotros mismos o desde donde los demás nos valoran, especialmente cuando no viene incorporado en nuestra manera de ser en la infancia o en la adolescencia. Según este escritor: "El nivel de nuestra autoestima no se consigue de una vez y para siempre en la infancia. Puede crecer durante el proceso de maduración o se puede deteriorar. Hay gente cuya autoestima era más alta a los diez años que a los sesenta, y viceversa. La autoestima puede aumentar y decrecer y aumentar otra vez a lo largo de la vida". Los adolescentes que consideran que tienen una autoestima elevada, consiguen afrontar las experiencias de manera más auto-realizante. (Dodgson y Wood, 1998; Tashakkori, Thompson, Wade y Valente, 1990) y (Di Paula y Campbell, 2002).

Trent, Russell y Cooney (1994) realizaron un estudio aplicando la escala SelfPerception Profile for Adolescents siguiendo el modelo de Auto concepto de Harter, que evalúa 8 aspectos del auto concepto: trabajo escolar, aceptación social, competencia atlética, apariencia física, atractivo romántico, rechazo entre sus amistades, competencia para el trabajo y una medida global para la capacidad de trabajo. Los resultados demostraron una gran consistencia de estas dimensiones analizadas y la multidimensionalidad de variables implicadas en el Auto concepto, ya confirmado por el modelo de Harter. Los estudios realizados por Harper y Marshall 
en 1991 (G. Sánchez, F. Jiménez y V. Merino, 1997) arrojaron que las chicas presentaban, significativamente, más problemas y niveles más bajos de autoestima que los chicos.

\subsubsection{La autoestima en la mujer}

Según Matud, M.P. (2004) "las mujeres con más confianza en sí mismas y las que se valoraban de forma más positiva tienen mejor salud, mostrando menos síntomas depresivos, de ansiedad, somáticos y disfunción social. Las mujeres que se valoraban de forma más negativa se caracterizan por tener un estilo de afrontamiento del estrés emocional y las mujeres con mayor confianza en sí mismas tenían un estilo de afrontamiento más racional y menos emocional".

Por otra parte, la autoestima en la mujer se manifiesta de forma diferente según la edad, siendo menor en la adolescencia y aumentando gradualmente a través del período adulto (Robins, Trzesniewski, Tracy, Gosling y Potter, 2002).

Branden, (2010) explica historias personales de mujeres que, tras luchar por su autoestima, se han transformado y han construido vidas sobre los citados pilares de la autoconciencia, la auto aceptación, la autorresponsabilidad, la autoafirmación, la determinación y la integridad personal. Además, explica cómo las mujeres pueden cultivar esas virtudes básicas para alcanzar su pleno potencial y adoptar estrategias efectivas que ayuden a resolver problemas muy comunes, desde convencer a los miembros de la familia para que colaboren en las tareas domésticas hasta poner fin a los comentarios sarcásticos de un compañero sentimental. Branden muestra las cuestiones que afectan especialmente a las mujeres, desmitifica las creencias clásicas sobre el amor romántico o el concepto de éxito y recuerda que la autoestima no es un don que venga dado por los demás, sino una actitud que consiste en romper con los malos hábitos y dar los pasos necesarios para crearnos una vida más satisfactoria.

Marcela Lagarde (2018) expone el paradigma feminista de principio del presente milenio con una perspectiva macrosocial. Considera que la autoestima en la mujer se ve afectada por factores culturales, sociales y políticos. En su libro Claves feministas para la autoestima de las mujeres, refleja que la mejora de la autoestima en la mujer se puede hacer de forma individual, pero implica a todas las mujeres y debe ser tratado tanto personal como políticamente, ya que es necesario hacer cambios sociales y culturales complejos para desarticular la construcción de género contemporánea. "Cada mujer requiere revisar su auto identidad desde una perspectiva feminista para hacer coincidencia de sus claves de autoestima".

\section{MARCO PRÁCTICO}

\subsection{Descripción de la muestra}

La población inicial está integrada por 14 niñas adolescentes jugadoras de fútbol, todas ellas integrantes del equipo de fútbol de Pozoblanco (Córdoba) cadete $B$, con edades comprendidas entre los 10 y 15 años. Para la selección de casos se fijaron 
como criterios de inclusión pertenecer al Club de fútbol Pozoblanco y tener edades comprendidas entre 11 y 15 años.

En el estudio, han participado 13 de las jugadoras del grupo de edad específico adolescentes entre 11 a 15 años, entre los cursos escolares de $6^{\circ}$ de Primaria y $4^{\circ}$ de la ESO, acorde al trabajo de investigación. Es una muestra intencional.

De ellas participan 10 en el programa, con diferente nivel de asistencia, ya que tres de ellas sólo van al taller de presentación y deciden no participar en el programa. Finalmente, el número de participantes real en el estudio asciende a un total de 10. La muestra representa el $71 \%$ de la población total inicial de jugadoras del equipo de fútbol de Pozoblanco (Córdoba) en la categoría de cadete B. Las integrantes de la muestra residen en Pozoblanco y otros municipios cercanos, todas ellas localidades de la provincia de Córdoba.

\begin{tabular}{|c|c|}
\hline$n$ & edad \\
\hline 1 & 10 \\
\hline 1 & 11 \\
\hline 3 & 12 \\
\hline 5 & 13 \\
\hline 1 & 14 \\
\hline 3 & 15 \\
\hline
\end{tabular}

Figura. 4: Composición edades de la población inicial.

Fuente: Elaboración propia.

En cuanto al tiempo practicando fútbol y la posición en el equipo:

\begin{tabular}{|l|l|l|l|}
\hline \multicolumn{2}{|c|}{$\begin{array}{c}\text { Tiempo } \\
\text { practicando }\end{array}$} & $\mathbf{n}$ & \multicolumn{2}{c|}{ Posición } & $\mathbf{n}$ \\
\hline 0-1 año & 3 & Portera & 1 \\
\hline 2-4 años & 4 & Defensa & 8 \\
\hline 5-7 años & 3 & Centro & 3 \\
\hline 8 más años & 4 & Delantero & 4 \\
\hline
\end{tabular}

$\left.{ }^{\star}\right)$ algunas jugadoras tienen varias posiciones

Figura 5: Antigüedad practicando y posiciones ocupadas de la muestra.

Fuente: Elaboración propia.

En relación con otros deportes, aficiones y talentos que reconocen, 4 practican pádel, 4 baile, 2 baloncesto, 1 taekwondo, 1 atletismo, 1 balonmano, 1 tenis, 1 montar a caballo, 1 running y 2 deportes en general. Entre las aficiones favoritas 4 consignan en la encuesta realizada salir, 2 escuchar música, 1 cantar, 1 jugar a 
videojuegos, 1 carnaval, 1 ver Netflix, 1 comer, 1 ser cómico y 1 tocar el piano. El equipo juega en la categoría $2^{\underline{a}}$ Cadete femenina de Córdoba.

La muestra seleccionada representa aproximadamente el $10 \%$ del total de las niñas jugadoras de fútbol cadetes de la provincia de Córdoba.

\subsection{Diseño y tipo de investigación}

\section{Diseño de investigación}

Es experimental, orientado a determinar la adecuación o no de nuestra hipótesis principal a la realidad, aplicando un conjunto de talleres digitales a la muestra seleccionada, constituida por un único grupo de investigación. Esto representa la no existencia de grupo control.

Tipo de investigación

Trabajo experimental de campo, con un enfoque de tipo investigación de acción participativa (PAR): un enfoque de investigación que ensalza tanto la participación como la acción, enfatizando la investigación colectiva y la experimentación. Ezequiel Ander-Egg (2003), hace una breve referencia a la aparición y desarrollo de la Investigación de acción participativa (IAP) como propuesta metodológica de gran valía. La investigación es de tipo longitudinal, ya que se toman datos antes y después de la intervención, incluyendo, además, el análisis correspondiente sobre si la actuación realizada determina la consecución de los objetivos.

\subsection{Descripción del programa}

El programa implementado, consta de 6 talleres ejecutados dirigidos a desarrollar actividades cuyo foco es la sensibilización, conocimiento y fortalecimiento de la autoestima en adolescentes deportistas.

El programa, denominado 4Adelante, se desarrolló desde el 10 de marzo de 2020 hasta el 11 de junio de 2020. Los días seleccionados para su implementación fueron los jueves, si bien el programa contempló también los espacios inter-talleres para el desarrollo de actividades a nivel individual.

\subsubsection{Ejecución, fases y actividades del programa}

Desde diciembre 2019 hasta julio 2020 se diseña e implementa el programa, en paralelo al trabajo de investigación teórico. Durante este tiempo y con la posterior extracción de conclusiones, se han generado gran cantidad de actividades, recogidas en tres fases:

1. Preparación del programa.

2. Desarrollo del programa.

3. Extracción de conclusiones.

\section{Fase 1: preparación}

En esta fase se desarrollan las siguientes actividades:

- Contacto con dirigentes del Club Pozoblanco.

- Preparación de autorizaciones y carta descriptiva. 
- Creación de cuenta específica de correo electrónico.

- Entrega de autorizaciones y carta descriptiva.

- Recogida de autorizaciones.

- Preparación de los talleres presenciales.

De manera paralela a las actividades relacionadas anteriormente, el equipo de investigación se reunió presencialmente en numerosas ocasiones para:

- Establecer la duración del desarrollo del programa en 3 meses.

- Determinar el número de talleres a realizar. Se decidió hacer un total de 6 talleres. Centrados cada uno en un pilar escogido de los 6 referidos por Braden, con una cadencia quincenal.

- Taller 0: Presentación del programa y determinación inicial de los niveles de autoestima individuales, a través del test de Rosenberg.

- Taller 1: Autoconocimiento.

- Taller 2: Auto aceptación.

- Taller 3: Auto respeto.

- Taller 4: Autoconfianza.

- Taller cierre: Extracción de conclusiones y aplicación posterior del test de Rosenberg.

- Realizar una ficha control de cada uno de los talleres

- Desarrollar cada uno de los talleres presenciales, acorde a las fichas control ya citadas. Se prepara en cada caso una presentación de power point, con una parte teórica y uno o varios videos a comentar y una parte lúdica e interactiva, utilizando diferentes herramientas. Se diseñan juegos asociados al contenido de cada taller.

- Diseñar una encuesta inicial para el taller de inicio

- Trasladar a la herramienta Survey Monkey el test de Rosenberg, tanto inicial como final.

\section{Creación del imagotipo}

Se decidió crear un imagotipo como representación gráfica de la marca, que representaría el programa en su conjunto. Como icono se seleccionó un balón de fútbol, debido a la actividad desarrollada por las niñas y como nombre de la marca: Adelante, representando el lema que debería trascender de la práctica del programa de fortalecimiento de la Autoestima. Además, se incorporó delante un 4 unido a la $\mathrm{A}$ de ADELANTE resaltando 4A, en base a los 4 talleres presenciales, que todos ellos empiezan por A: Autoconocimiento, Auto aceptación, Auto respeto y Autoconfianza. Se combinan el icono y el texto en una única composición.

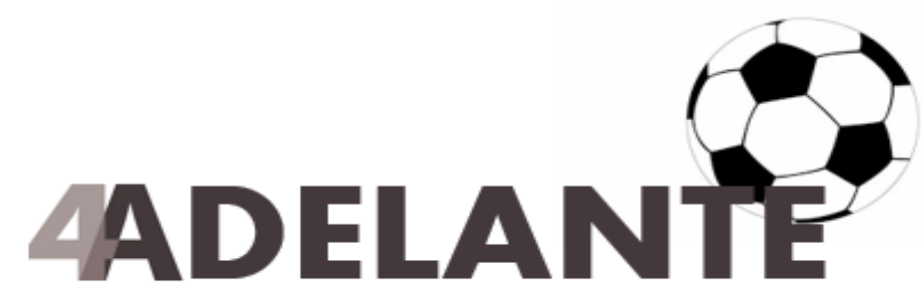

Figura 6: Logotipo del programa.

Fuente: Elaboración propia. 


\section{Fase 2: ejecución.}

Una vez realizada la preparación de los talleres, se inicia el desarrollo de los mismos.

Desarrollo del taller 0: Autoestima.

El taller de iniciación del programa para el fortalecimiento de la autoestima de las jugadoras de fútbol, denominado taller 0 , se realiza el 10 de marzo. Se utilizó para "romper el hielo", explicar el formato del mismo, introducir al equipo en el concepto de autoestima y resolver dudas. Se realizó el test de Rosenberg y la encuesta diseñada por el equipo para obtener datos personales, direcciones, cuentas de correo electrónico y teléfono de contacto de las mismas. Al ser un taller presencial asiste de nuevo Paula Salado.

El día 13 de marzo de 2020 se decretó el estado de alarma en el país por el COVID19, lo que supuso el cese de los entrenamientos y días después, el confinamiento de toda la población del país. Esta situación comprometió la implementación de los talleres tal y como se habían diseñado. El mantenimiento en el tiempo de dicho estado de confinamiento, nos llevó a plantear diferentes escenarios sobre la continuidad del proyecto. Las alternativas fueron: cambiar el tema de investigación, hacer sólo un estudio descriptivo (sin implementación del programa) o rehacer los talleres en un formato completamente digital. Pese al reto personal que suponía la adaptación de todos los talleres y a que ya estuviesen desarrollados en su formato presencial, se decidió la reconversión de los que estaban pendientes de implementación a un formato digital y en remoto.

Esto nos llevó de nuevo a una fase de preparación, con la adaptación de los talleres a la modalidad online, remodelación de algunos contenidos y búsqueda de nuevas herramientas interactivas: se escogió Edmodo por tratarse de una plataforma tecnológica, social, educativa, que permite la comunicación entre los alumnos y los profesores en un entorno cerrado y privado, de uso en la nube, al mismo tiempo que permite a los padres, que así lo deseen, monitorizar las tareas de sus hijas y, además, era gratuita. Brindaba un entorno intuitivo y amigable y seguro, en el que se pueden compartir mensajes, archivos y enlaces, un calendario de trabajo, así como proponer tareas y actividades y gestionarlas. El profesor puede ver todo lo que se publica en Edmodo y validarlo antes. Igualmente, posibilitaba hacer una estructuración de los talleres por clases, disponer de una biblioteca y crear una estructura de carpetas para asociar y clasificar la documentación de cada uno de los talleres.

Se decidió utilizar Zoom como herramienta para impartir las sesiones de los jueves en remoto y la plataforma Edmodo para soportar la documentación, actividades entre talleres y favorecer la propia adherencia al programa por parte de las jugadoras. Se escogieron también otras herramientas como Kahoot, Klaxoon, Survey Monkey, cuestionarios asociados a la plataforma Edmodo, así como el grupo de Whatsapp, para dinamizar las actividades de los talleres. 


\section{Fase 3: Extracción de conclusiones y propuesta de continuidad.}

Tras la realización del taller de cierre, el equipo se reúne de nuevo de manera virtual para extraer las conclusiones de los talleres y del programa global, desde diferentes perspectivas:

- Niveles de autoestima finales vs iniciales (tanto a nivel individual como grupal).

- Feed-back de los talleres, dado por las jugadoras el último día.

- Análisis de asistencias y participaciones.

- Análisis de la percepción de las participantes y comparativa de la percepción con los resultados de Rosenberg.

- Análisis cualitativo de actitudes y comportamientos reflejados en los vídeos.

- Redacción de conclusiones.

- Propuesta de continuidad.

\subsubsection{Materiales}

Se han desarrollado varios materiales digitales para el desarrollo online.

\section{$\underline{\text { Test de Rosenberg en Survey Monkey }}$}

Dada la edad de la muestra se digitaliza el Test de Rosenberg y se hace a las jugadoras a través de Survey Monkey, desde sus dispositivos móviles, desde el taller inicial, aunque se desarrolló de manera presencial. Se desarrolla de igual manera en el taller de cierre.

\section{Encuesta equipo}

Se desarrolla por parte del equipo una encuesta con datos personales con el objetivo de que cada participante la conteste el día de presentación de los talleres (taller 0), se hace también a través de Survey Monkey y recoge los siguientes ítems:

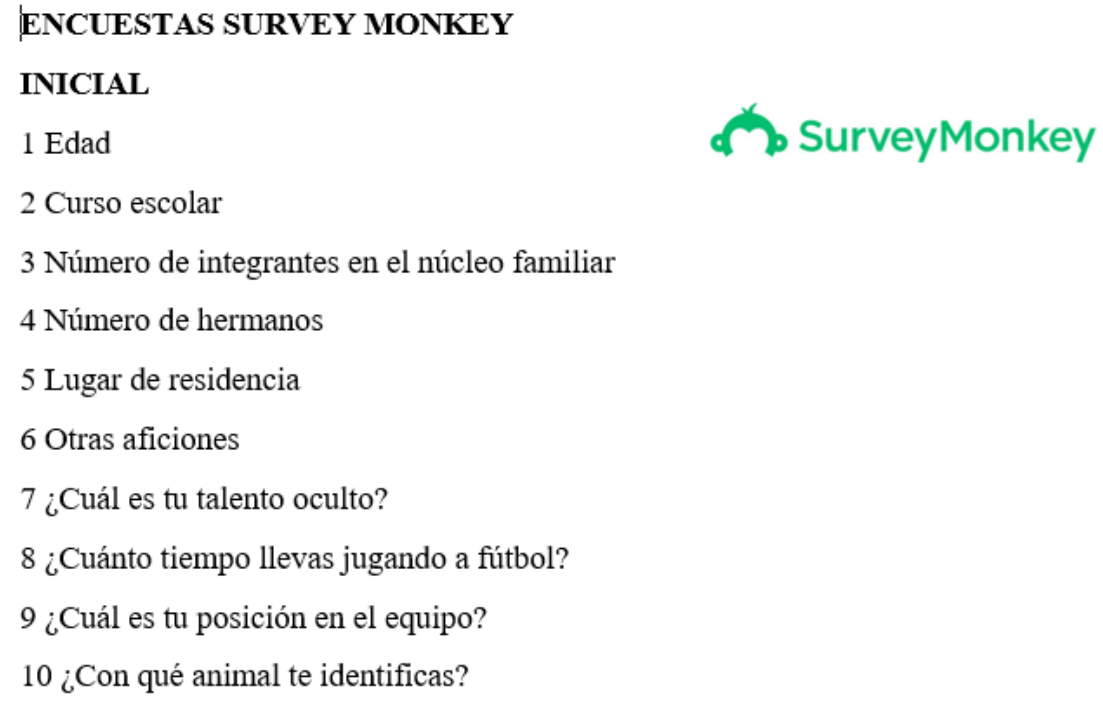

Figura. 7: Encuesta Survey Monkey inicial de datos personales.

Fuente: Elaboración propia. 
Test de Feedback de los talleres

\section{ENCUESTAS SURVEY MONKEY \\ FEEDBACK FINAL}

\section{SurveyMonkey}

1- ¿Qué te ha parecido el contenido de los talleres?

2- ¿Crees que el $\mathrm{n}^{\circ}$ de talleres es el adecuado?

3- ¿Cómo te ha parecido la dinamización de los talleres?

4- ¿Recomendarías a una amiga este programa de talleres?

5- ¿Prefieres los talleres presenciales o digitales?

6- ¿Cuánto te ha servido el uso de herramientas digitales durante los talleres: Zoom, Edmodo, Kahoot, Klaxoon, Survey Monkey?

7- ¿Qué cambiarías /añadirias/quitarías de estos talleres?

8- Según tu apreciación, ¿cómo ha cambiado tu nivel de autoestima?

9- De los 4 Talleres: Autoconocimiento, Autoaceptación, Autorespeto y Autoconfianza ¿Cuál has disfrutado más?

10- Si tuvieses una amiga con baja autoestima ¿qué le dirías de todo lo que has aprendido?

Figura. 8: Encuesta Survey Monkey feedback de los talleres.

Fuente: Elaboración propia.

\section{Presentación de cada taller}

Se realizó una presentación de PowerPoint que se utilizó en cada una de las sesiones (tanto la presencial como las sesiones digitales), recogiendo conceptos, dinámicas y actividades a realizar. Se compartió en cada sesión con todas las niñas a través de Zoom. Luego fueron colgadas en Edmodo para facilitar el acceso a las mismas.

Contenido digital incluido en la plataforma Edmodo.

Se dieron de alta cada uno de los talleres en la plataforma educativa Edmodo, incorporando una biblioteca estructurada con todo el material asociado a cada uno de ellos. Se crearon una serie de actividades vinculadas a cada taller para que las niñas las desarrollaran durante la sesión por Zoom o entre sesiones. Previa a la sesión por Zoom, se dio acceso a todas las participantes al taller creado en Edmodo. Y al finalizar la misma, se difundió en el muro de la plataforma una publicación compartida con la presentación vista durante la sesión de Zoom y se vinculó el acceso a los videos de las sesiones con Google Drive.

Ficha de observación.

Se realiza tras la finalización de cada taller. Recoge de cada uno las emociones expresadas por las participantes en el mismo, valoración de la evolución actitudinal y gestual en el proceso, a lo largo del tiempo.

Diplomas.

Tras la finalización del taller $\mathrm{n}$ 눙, se envía un diploma de agradecimiento a cada jugadora, en nombre de los 4 investigadores. Cada diploma está ilustrado con el animal con el que cada una de ellas se identificaba y que quedó recogido en la encuesta del primer día. 


\section{RESULTADOS DE LA APLICACIÓN DEL PROGRAMA}

\subsection{Resultados de la aplicación de la escala de Rosenberg}

A continuación, presentamos el resultado del test de Rosenberg aplicado a la muestra en el taller inicial (0) y el final (6) por integrante de la muestra y el promedio del equipo. Cada una de las participantes está identificada mediante el animal con el que sentía representada y que cada una eligió en el test inicial desarrollado en el taller 0 .

\begin{tabular}{|c|c|c|c|}
\hline \multicolumn{4}{|c|}{ Test de Rosenberg Marzo } \\
\hline Jugadoras & Preguntas & Puntuación Total & Resultado \\
\hline 1 & Koala & 27 & Autoestima saludable \\
\hline 2 & Leona & 20 & Autoestima saludable \\
\hline 3 & Loro & 27 & Autoestima saludable \\
\hline 4 & Cabra & 18 & Autoestima saludable \\
\hline 5 & Guepardo & 13 & Autoestima muy baja \\
\hline 6 & Gallo & 16 & Autoestima saludable \\
\hline 7 & Lobo & 14 & Autoestima muy baja \\
\hline 8 & Gato & 16 & Autoestima saludable \\
\hline 9 & Serpiente & 27 & Autoestima saludable \\
\hline 10 & Perro & 11 & Autoestima muy baja \\
\hline 11 & Tigre & 27 & Autoestima saludable \\
\hline 12 & Caballo & 25 & Autoestima saludable \\
\hline 13 & Perro Caniche & 24 & Autoestima saludable \\
\hline \multicolumn{2}{|c|}{ TOTAL } & 20,38 & Autoestima saludable \\
\hline
\end{tabular}

Figura. 9: Tabla de resultados del test de Rosenberg inicial.

Fuente: Elaboración propia.

\begin{tabular}{|c|c|c|c|}
\hline \multicolumn{4}{|c|}{ Test de Rosenberg Junio } \\
\hline Jugadoras & Animal & Puntuación Total & Resultado \\
\hline 1 & Koala & 28 & Autoestima saludable \\
\hline 2 & Leona & 28 & Autoestima saludable \\
\hline 3 & Loro & $\# N / D$ & $\# N / D$ \\
\hline 4 & Cabra & 14 & Autoestima muy baja \\
\hline 5 & Guepardo & $\# \mathrm{~N} / \mathrm{D}$ & $\# N / D$ \\
\hline 6 & Gallo & 16 & Autoestima saludable \\
\hline 7 & Lobo & 17 & Autoestima saludable \\
\hline 8 & Gato & 25 & Autoestima saludable \\
\hline 9 & Serpiente & 26 & Autoestima saludable \\
\hline 10 & Perro & $\# N / D$ & $\# N / D$ \\
\hline 11 & Tigre & 23 & Autoestima saludable \\
\hline 12 & Caballo & $\# N / D$ & $\# N / D$ \\
\hline 13 & Perro Caniche & 23 & Autoestima saludable \\
\hline \multicolumn{2}{|c|}{ TOTAL } & 22,22 & Autoestima saludable \\
\hline
\end{tabular}

Figura. 10: Tabla de resultados del test de Rosenberg final.

Fuente: Elaboración propia. 


\subsection{Análisis de los resultados obtenidos en la implementación}

Promedio de puntuación de Marzo VS Junio

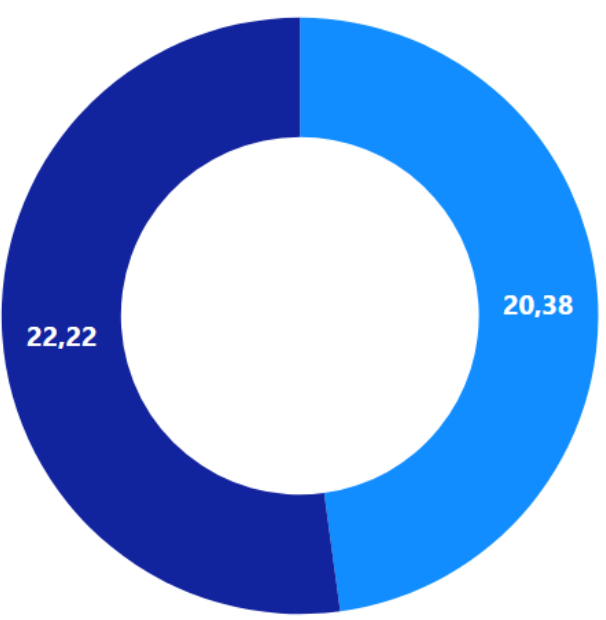

- Promedio Resultados de Marzo • Promedio Resultados de Junio

Figura 11: Diagrama comparativo de los niveles de autoestima.

Fuente: Elaboración propia.

Tras aplicar el test de Rosenberg en el taller 0 el resultado obtenido fue de 20,38 sobre 30, y transcurridos los talleres se aplicó nuevamente del test en la sesión 6 y el resultado fue de 22,22 sobre 30. Al comparar ambas mediciones de la autoestima de la muestra, observamos un aumento de la autoestima grupal de 1,84 puntos sobre 30 .

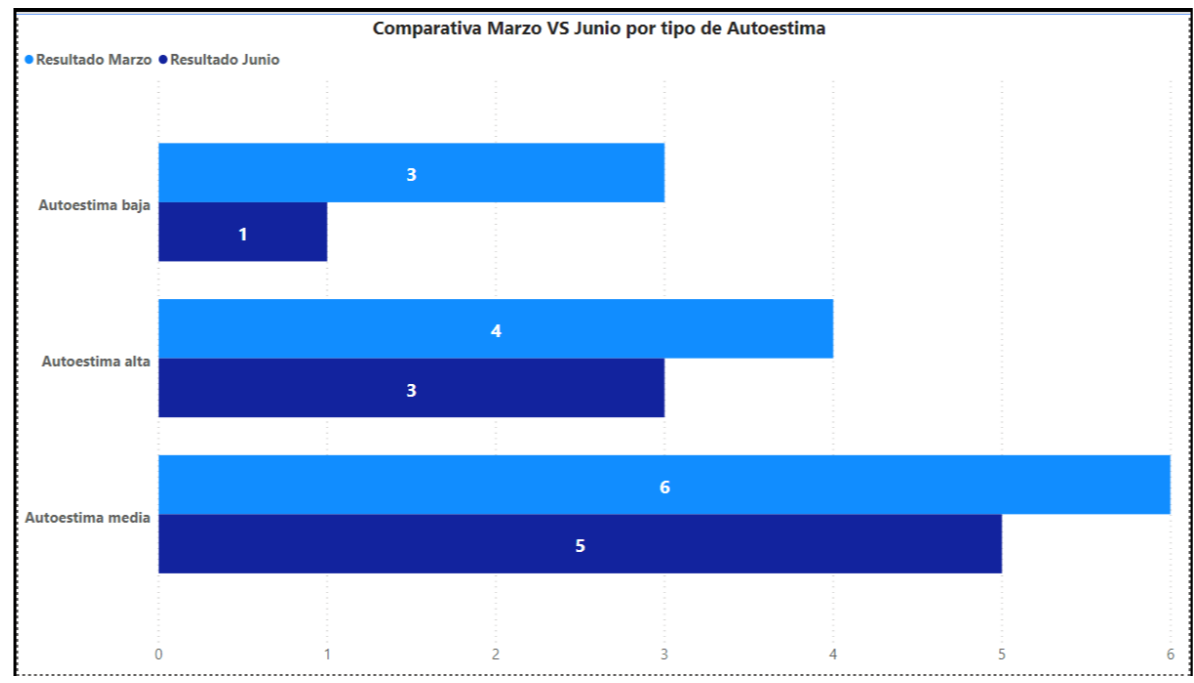

Figura. 12: Comparativa de autoestima marzo vs junio, según niveles.

Fuente: Elaboración propia

En este gráfico se representa el número de participantes que tenían diferentes niveles de autoestima (baja, media y alta) según Rosenberg, comparando los resultados de marzo y junio. Observamos que en marzo 3 de ellas tenían autoestima 
baja y solamente una en junio. 4 tenían autoestima alta en marzo y 3 en junio. Por último, 6 jugadoras con autoestima media en marzo y 5 en junio.

Aquí cabe destacar:

- $\quad$ Que no todas las participantes hicieron el test final

- De las que lo hacen falta concluir respecto a las que lo han hecho en marzo y junio que resultados se obtiene)

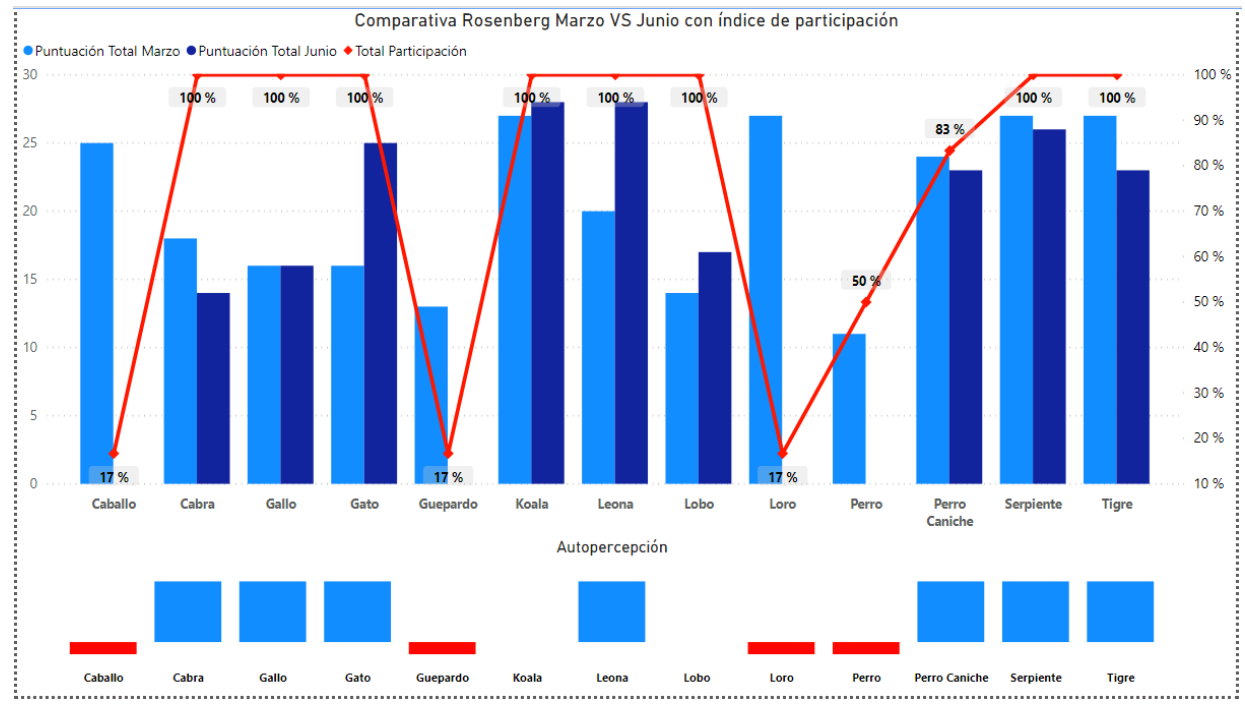

Figura. 13: Comparativa de autoestima marzo vs junio, según participación.

Fuente: Elaboración propia.

Este gráfico representa la diferencia en el nivel de autoestima de cada una de las integrantes de la muestra incluyendo también el porcentaje de asistencia a los talleres del programa 4Adelante. También incluimos como comparativa, la percepción que cada una tiene de cómo ha evolucionado o no su nivel de autoestima. En el taller de cierre se realiza una encuesta cualitativa en la que se les pregunta sobre como creían que se había modificado su nivel de autoestima tras la realización de los talleres. Las barras de color rojo de la parte inferior representan a las niñas que no han realizado el test; el color azul representa a las que creen que su autoestima sí ha cambiado; las barras que no tienen ningún color corresponden con las participantes que creen que su autoestima no ha cambiado desde marzo.

\section{CONCLUSIONES}

\subsection{Conclusiones acerca de la hipótesis general}

Después de aplicar el programa 4Adelante y realizar el trabajo de investigación, a raíz de los test de Rosenberg realizados al principio y final del programa, se observó una mejora de la autoestima a nivel grupal de la muestra. Por otro lado, los resultados de la encuesta realizada a las participantes arrojan las mismas conclusiones.

Con todo esto se afirma la hipótesis general planteada en el proyecto de investigación: "la aplicación de un programa para potenciar la autoestima en adolescentes, jugadoras de fútbol, incrementará el nivel de autoestima de las mismas". 


\subsection{Conclusiones acerca de las hipótesis específicas}

En relación a la hipótesis específica 1, se confirma la necesidad de medida de manera previa del nivel de autoestima de la muestra para conocer el punto de partida antes de la ejecución del programa.

Al aplicar un programa de fortalecimiento de la autoestima, ésta se ha visto aumentada tras su participación en los talleres y actividades del programa por parte de las adolescentes. Con esto queda afirmada la segunda hipótesis específica.

Es imprescindible realizar el test al final del programa para poder evaluar de una forma objetiva la evolución de la autoestima en las participantes tras la aplicación del programa de talleres. De otra forma sólo tendríamos la valoración subjetiva de las participantes en el test final, que no nos proporcionaría la objetividad necesaria al extraer las conclusiones. Con esto queda validada la hipótesis específica 3.

\subsection{Conclusiones a cerca del objetivo general}

La adolescencia es una etapa de grandes cambios y que marca la importante transición entre la niñez y la edad adulta. Son años de transición en los que los jóvenes comienzan a perfilar su personalidad y que, en ocasiones, pueden ser muy complicados, no solo para ellos, sino también para su entorno. El diseño de los talleres ha tenido en cuenta estos aspectos y se ha regido además por la importancia del desarrollo de la autoestima si además son niñas y practican un deporte que puede ser considerado eminentemente masculino.

Fomentar su autoestima es clave para su bienestar psicológico, algo que determinará su edad adulta a nivel emocional. Pero ¿cómo ayudarles en este proceso?, trabajando la autoestima, seleccionado pilares clave de la misma y desarrollando un programa acorde a los mismos.

Los pilares a los que Branden hace referencia en su libro: "Los seis pilares de la autoestima", nos han servido de inspiración para el desarrollo del programa 4Adelante. Al implementar el programa se concluye la importancia de desarrollar actividades de carácter lúdico para favorecer el aprendizaje. El contenido que inicialmente era mayoritariamente digital y se convirtió en $100 \%$ tras la reformulación de los talleres, ha fomentado la participación y la adherencia al programa.

Se identifica como conclusión, que se ha creado un programa para desarrollar la autoestima, específico, para jugadoras de fútbol adolescentes.

\subsection{Conclusiones acerca de los objetivos específicos}

1. En relación al objetivo específico 1, se concluye que es muy importante realizar una medición inicial objetiva de los niveles de autoestima antes de la implementación del programa.

2. Aplicación del programa online diseñado: 4Adelante

3. Inicialmente se había planteado talleres presenciales, aunque a raíz de la situación del COVID19 se han tenido que realizar on-line y complementar con actividades digitales. 
4. Determinar el nivel de autoestima del equipo después de la implementación del programa.

\section{PRÓXIMAS LÍNEAS DE INVESTIGACIÓN}

Creemos que sería oportuno trabajar los pilares incluidos en el programa 4Adelante con mayor profundidad y muy probablemente con mayor número de sesiones, se haría necesaria la continuidad del proyecto en el propio club, para que se beneficien del mismo de la forma más eficaz posible.

De igual modo entendemos que se puede extrapolar a los demás equipos de jugadoras cadetes, ya que el programa está muy orientado a las edades incluidas en esta categoría y equipos femeninos, al orientarse especialmente a la situación que puedan vivir las niñas y en el fútbol.

Proponemos para preparar futuros programas tener en cuenta los resultados obtenidos tras la evaluación final, para ver desde el feedback dado, posibles mejoras a incorporar.

En cualquier caso, nuestra propuesta incluiría como puntos importantes:

- Incrementar el número de talleres totales.

- Establecer un programa base híbrida, presencial/online, que pueda adaptarse según la realidad del momento o del equipo.

- Utilizar un espacio de tiempo del taller 0 destinado a la formación en el manejo de las herramientas a utilizar.

- Que una de las futuras líneas de actuación sea trabajar más profundamente el taller de autoconfianza.

- Dedicar más tiempo al final de cada taller para hacer emerger voluntariamente situaciones personales.

La propuesta de continuidad sería adaptar el programa y formar a los entrenadores y al club para que pudieran desplegarlo a la población que no ha participado y a nuevas incorporaciones y hacerlo extensivo a otros clubes de la provincia de Córdoba.

Otra propuesta de cara al futuro es el desarrollo de un programa similar a 4Adelante, encaminado al fortalecimiento de la autoestima en equipos cadetes femeninos, adolescentes, en otros deportes. Entendemos que es extrapolable también a equipos masculinos, con la correspondiente adaptación de los talleres, así como a otras zonas geográficas, tanto en el ámbito rural como en el urbano.

\section{REFERENCIAS}

Abbassi, E., Tutora, P., \& Pacheco, C. R. (2015). "Prejuicio de los hombres hacia las mujeres que practican deportes de contacto ".

https://riull.ull.es/xmlui/bitstream/handle/915/3219/Prejuicio+de+los+hombres+haci $\underline{a+l a s+m u j e r e s+q u e+p r a c t i c a n+d e p o r t e s+d e+c o n t a c t o+. p d f ? \text { sequence }=1}$ 
Ander-Egg, E. (2003). Repensando la investigación-acción-participativa. http://www.academia.edu/download/54884819/E Ander Egg CAPITULOS 2 Y 4.pdf

Augusto Ceballos-Ospino, G., Paba-Barbosa, C., Suescún, J., Celina Oviedo, H., Herazo, E., \& Campo-Arias, A. (2017). Validity and Dimensionality of the Rosenberg Self-esteem Scale Among College Students. Pensamiento Psicológico, 15(2), 29-39. https://doi.org/10.11144/Javerianacali.PPSI15-2.vdea

Autoestima, N. B.-L. seis pilares dela, \& 1995, undefined. (n.d.). Desarrollo de la autoestima. In hermandadblanca.org. Retrieved May 15, 2020, from https://hermandadblanca.org/wpcontent/uploads/2016/03/hermandadblanca org desarrollo-de-la-autoestima.pdf

Bonet, J. (1997). Sé amigo de ti mismo: manual de autoestima. https://books.google.es/books?hl=es\&lr=\&id=iTwVTnXcuBcC\&oi=fnd\&pg=PA11\&d $\mathrm{q}=$ Bonet+manual+de+autoestima\&ots=uOIQyGk7nr\&sig=-6oDtgWObQIEzu41JwzRTE9s w

Braden, N. (1995). LOS SEIS PILARES DE LA AUTOESTIMA. Nathael Braden 1.-. Academia, Edu, 1-6.

Branden, N. (2010). La autoestima de la mujer.

Brown, J. D., Dutton, K. A., \& Cook, K. E. (2001). From the top down: Self-esteem and self-evaluation. Cognition and Emotion, 15(5), 615-631. https://doi.org/10.1080/02699930126063

Cardinal, C. C.-C., autoestima, L. diez mandamientos de la, \& 2007, undefined. (n.d.). Los diez mandamientos de la autoestima.

com/libros-on-line/category/marcela-lagarde, M. L.-. weebly., \& 1999, undefined. (n.d.). Claves feministas para liderazgos entrañables. We.Riseup.Net. Retrieved May 29, 2020, from https://we.riseup.net/assets/119766/Claves+feministas+para+liderazgos+entrañab les.docx

Company, S. C.-H. F. and, \& 1967, undefined. (n.d.). The antecedents of selfesteem San Francisco.

del, F. C.-M. en P. de la A. F. y, \& 2010, undefined. (n.d.). Comparación de los estados de ánimo antes y después del partido entre dos equipos de fútbol.

Diferencias en la calidad psicométrica de test construidos mediante la estrategia pedagógica audiovisual y las estrategias pedagógicas tradicionales. (n.d.). Revistaespacios.Com. Retrieved May 22, 2020, from https://www.revistaespacios.com/a18v39n25/18392507.html 
Elmi José Odor Hurtado, Pilar Portugal de la Cuerda, Paula Salado García y Olga Sanz Gómez

En, A., Mujer, L. A., De, U. A., Relevancia En, S. U., Salud, L. A., \& Matud, M. P. (2004). Avances en Psicología Latinoamericana. In revistas.urosario.edu.co (Vol. 22). https://revistas.urosario.edu.co/index.php/apl/article/view/1464

Ezequiel Ander-Egg, P. (n.d.). Repensando la Investigación-Acción Participativa.

Investigativas En Educación, A., Rica, C., Luisa, M., \& Pereira, N. (n.d.-a). Los contenidos de este artículo están bajo una licencia Creative Commons. Retrieved June 14, 2020, from http://revista.inie.ucr.ac.cr

Investigativas En Educación, A., Rica, C., Luisa, M., \& Pereira, N. (n.d.-b). Los contenidos de este artículo están bajo una licencia Creative Commons. In redalyc.org. Retrieved May 22, 2020, from http://revista.inie.ucr.ac.cr

Jack, C. (2008). Chicken soup for the soul 1.

James, W. (1890). The principles of Psychology.

Lagarde, M. (2018). Género y feminismo: desarrollo humano y democracia. https://books.google.es/books?hl=es\&lr=\&id=vwSzDwAAQBAJ\&oi=fnd\&pg=PT4\& $\mathrm{dq}=$ Marcela + Lagarde $+\% 22 \mathrm{Género}+\mathrm{y}+$ feminismo\%22\&ots=6pkPFkec3M\&sig=15v QRJEn8bVH-CTTpl8SZjjJoMs

Landivar, S. H.-U. R., Quetzaltenango, undefined, \& 2014, undefined. (n.d.). Nivel de autoestima y éxito deportivo en jóvenes que practican taekwondo"(estudio realizado con deportistas en área de competencias)(Tesis de grado).

Marcos, L. (2007). La autoestima: nuestra fuerza secreta. http://iesfcodeorellana.juntaextremadura.net/datos/orientacion/diego/educacionen-valores/autoestima/articulo-autoestima-de-lius-rojas-marcos.pdf

Mártir, V., \& Molina García, J. (2007a). BIENESTAR PSICOLÓGICO Y PRÁCTICA DEPORTIVA EN UNIVERSITARIOS. In European Journal of Human Movement (Vol. 18). https://www.redalyc.org/pdf/2742/274220374005.pdf

Mártir, V., \& Molina García, J. (2007b). BIENESTAR PSICOLÓGICO Y PRÁCTICA DEPORTIVA EN UNIVERSITARIOS. In European Journal of Human Movement (Vol. 18). https://www.redalyc.org/pdf/2742/274220374005.pdf

Maslow, A. (1991). Motivación y personalidad. https://books.google.es/books?h|=es\&lr=\&id=8wPdj2Jzqg0C\&oi=fnd\&pg=PR13\&d $\mathrm{q}=$ Teoria+de+la+motivacion+humana\&ots=Fi5ZqNfco\&sig=XDklIP9FR1aa4JIIXuYBeV8OdoA

Mckay, M., \& Fanning, P. (1991). Autoestima: evaluación y mejora.

McKay y Fanning. (1991). Autoestima: evaluación y mejora. 
Miguel, R., Zemel, M., (Rosario, L. C.-.., 2009), undefined, \& 2009, undefined. (n.d.). Procesos de aprendizaje sobre odontología legal en la formación odontológica de grado. Sedici.UnIp.Edu.Ar. Retrieved June 17, 2020, from http://sedici.unlp.edu.ar/handle/10915/75248

Miranda, C, \& Andrade, M. (2005). Influencia de la inteligencia múltiple, el currículo y la autoestima en el rendimiento académico.

Molina, J., Chorot, P., \& Valiente Bonifacio Sandín, R. M. (1993). Miedo a la evaluación negativa, autoestima y presión psicológica: Efectos sobre el rendimiento deportivo en adolescentes. Revistas.Um.Es, 14, 57-66. http://revistas.um.es/cpd

Morshead, C., Reynolds, B., Craig, C., Neuron, M. M.-, \& 1994, undefined. (n.d.). Neural stem cells in the adult mammalian forebrain: a relatively quiescent subpopulation of subependymal cells. Elsevier. Retrieved May 22, 2020, from https://www.sciencedirect.com/science/article/pii/0896627394900469

Naranjo, C. R., \& Caño González, A. (2012). Autoestima en la adolescencia: análisis y estrategias de intervención. In International Journal of Psychology and Psychological Therapy (Vol. 12). https://www.ijpsy.com/volumen12/num3/337/autoestima-en-la-adolescenciaanlisis-ES.pdf

Oteíza, E., Rodríguez, R., Carvajal, G., Carvajal, V., \& Sepúlveda, Y. (2011). Edición 12 Palabras clave: Autoconcepto Físico. In dialnet.unirioja.es (Vol. 8). https://dialnet.unirioja.es/servlet/articulo?codigo=3706586

Pastor, Y., Balaguer, I., \& García-Merita, M. L. (2003). El autoconcepto y la autoestima en la adolescencia media: Análisis diferencial por curso y género. Revista de Psicologia Social, 18(2), 141-159. https://doi.org/10.1174/021347403321645258

Paula, A. Di, social, J. C.-J. of personality and, \& 2002, undefined. (n.d.). Selfesteem and persistence in the face of failure. Psycnet.Apa.Org. Retrieved May 29, 2020, from https://psycnet.apa.org/record/2002-17813-015

pe Sánchez, G., Jiménez, F., \& Merino, V. (1997). AUTOESTIMA Y AUTOCONCEPTO EN ADOLESCENTES: UNA REFLEXIÓN PARA LA ORIENTACIÓN EDUCATIVA. In Revista de Psicología de la PUCP: Vol. XV. http://revistas.pucp.edu.pe/index.php/psicologia/article/view/5958

Pere, J., \& Barceló, R. (2017a). El deporte y la autoestima como factores de socialización e inclusión social. https://dspace.uib.es/xmlui/handle/11201/3843

Pere, J., \& Barceló, R. (2017b). El deporte y la autoestima como factores de socialización e inclusión social. https://dspace.uib.es/xmlui/handle/11201/3843 
Resolución, E. LA, Ansiedad Y Depresión, C. DE, \& Ma Bastida de Miguel, A. (2010). Importancia de aplicar terapias psicológicas de tercera generación the importance of applying third-wave psychological therapies when resolving a case of comorbid fibromyalgia with symptoms of anxiety and depression. www.interpsiquis.com

Ricardo Contreras, O., Gregorio Fernández, J., Miguel García, L., Palou, P., \& Ponseti, J. (2010). EL AUTOCONCEPTO FÍSICO Y SU RELACIÓN CON LA PRÁCTICA DEPORTIVA EN ESTUDIANTES ADOLESCENTES. In redalyc.org (Vol. 19, Issue 1). https://www.redalyc.org/pdf/2351/235116414002.pdf

Robins, R. W., Trzesniewski, K. H., Tracy, J. L., Gosling, S. D., \& Potter, J. (2002). Global Self-Esteem Across the Life Span. Psycnet.Apa.Org. https://doi.org/10.1037/0882-7974.17.3.423

Rosenberg, M. (1989). Society and the adolescent self-image. Revised edition.

Sanchez, T. (n.d.). Qué es la autoestima: definición, significado y tipos de autoestima. Retrieved May 22, 2020, from https://www.diariofemenino.com/articulos/psicologia/autoestima/que-es-laautoestima-definicion-significado-y-tipos-de-autoestima/

Steinberg, L., \& Morris, A. S. (2001). Adolescent Development. Annual Review of Psychology, 52(1), 83-110. https://doi.org/10.1146/annurev.psych.52.1.83

Trent, L. M. Y., Russell, G., \& Cooney, G. (1994). Assessment of self-concept in early adolescence. Australian Journal of Psychology, 46(1), 21-28.

https://doi.org/10.1080/00049539408259465

Trzesniewski, K. H., Donnellan, M. B., \& Robins, R. W. (2003). Stability of SelfEsteem Across the Life Span. Journal of Personality and Social Psychology, 84(1), 205-220. https://doi.org/10.1037/0022-3514.84.1.205

Twenge, J. M., \& Campbell, W. K. (2001). Age and Birth Cohort Differences in SelfEsteem: A Cross-Temporal Meta-Analysis Article in Personality and Social Psychology Review A Psychological Exploration of Engagement in Geek Culture View project Narcissism(s) and self-esteem(s) in cross-cultural context View project. Journals. Sagepub.Com, 5(4), 321-344. https://doi.org/10.1207/S15327957PSPR0504 3

\section{AUTORES}

Elmi José Odor Hurtado.

EAE business School. España.

\section{Pilar Portugal de la Cuerda.}

EAE business School. España.

\section{Paula Salado García.}

EAE business School. España.

\section{Olga Sanz Gómez.}

EAE business School. España. 